\title{
Rate-Equation Analysis for a Coupled-Cavity Laser with MMI Anti-Phase Coupler
}

\author{
Daan Lenstra \\ Photonics Integration Group, Dept. of Electrical Engineering, Eindhoven University of Technology, \\ P.O.Box 513, 5600MB Eindhoven, The Netherlands \\ d.lenstra@tue.nl
}

Keywords: semiconductor laser, coupled-cavity laser, multi-mode coupler, rate equations

\begin{abstract}
Rate equations are derived for the coupled-cavity laser with a multimode-interference coupler. A strategy and scheme is indicated for iterative self-consistent numerical solution of the steady-state equations. The presence of the linewidth-enhancement parameter is explicitly taken into account. Locking in stable single-mode anti-phase operation is numerically demonstrated and locking ranges are given. Numerical results are given for the output power and the operation frequency as functions of the pump strengths of the individual lasers. The shapes of output-intensity curves agree well with measured curves.
\end{abstract}

\section{Introduction}

A coupled-cavity laser (CCL) based on anti-resonant imaging in a multi-mode interference (MMI) coupler has been demonstrated in 2015 as a photonic integrated circuit [1]. This has revived interest in coupled-cavity lasers as widely tunable lasers for spectroscopy, sensing and other applications. The specially designed MMI anti-phase reflective coupler is based on a $3 \times 3$ reflective MMI coupler, described in [1], which leads to maximized coupling when the two lasers operate with 180 degrees phase difference. In this way, a self-stabilizing coupled device has been realized, unlike former devices based on air gaps [2,3], $2 \times 2$ couplers [4] and other principles $[5,6,7]$.

A dynamical theory for the CCL with MMI anti-phase coupler is not available yet. Many approaches are based on linear monochromatic analyses [2, 4, 5, 6, 7] and although this does yield insight into the coupled-mode operation of these devices, the dynamical process leading to frequency locking of the two constituent lasers requires analysis of coupled rate equations for the complex fields and the inversions in the two constituent lasers. Existing rate-equation theories for CCL's are for conventional couplings, i.e. passive dielectric slabs without multi-mode interference imaging. Ref. [7] first determines the modes of the coupled-cavity system and formulates for these modes photon rate equations, coupled to rate equations for the inversions in each cavity section. This approach gives no clue as to the dynamics of the locking process of the two constituent lasers. In the theory of ref. [8] local rate equations are formulated for the fields in each separate laser of the coupled system. This theory therefore does allow analysis of the evolution towards stable mutual locking.

Here we develop a rate-equation model much in the spirit of ref. [9] for semiconductor lasers with quantum-well active material and coupled through an MMI anti-phase reflector. In our theory, the dispersive role of the self-phase modulation parameter $\alpha$ is explicitly dealt with. The results of the analysis for stable steady-state operation will be presented and discussed. The theory is selfconsistent in the sense that the effective coupling coefficients depend on the respective inversions in each laser, where the latter in their turn depend on the effective coupling. This requires a selfconsistent solution, which is obtained by an iteration method.

The "knobs" of the coupled system are the injection currents of the two constituent lasers and the frequency difference (or detuning) of the two individual single laser modes. The theory describes if, why and how, due to the coupling, the two original isolated modes will combine to one single "super-mode", a situation referred to as locked state. We have found that for all practical values of $\alpha$ stable locking occurs for detuning values not exceeding $~ 3 \mathrm{GHz}$. A sizeable detuning interval of 
locking is important, as this allows proper fine tuning of the laser system, a feature fully employed in ref. [1] by using a phase modulator integrated in one of the cavities.

\section{Description of the model and derivation of rate equations}

We assume that two Fabry-Pérot lasers are very similar except that they differ 5-10\% in length and are coupled by a reflective MMI anti-phase coupler. This is schematically indicated in Fig.1, where the amplitudes $E_{1}$ and $E_{2}$ are evaluated at the points where each respective laser touches the MMI-coupler. Note that the coupling parameters have been designed in such a way that $C_{\mathrm{bar}}$ and $C_{\mathrm{x}}$ are 180 degrees out of phase and the sum of their absolute values equals unity. This is a property of the $3 \times 3$ reflective coupler in which only the two outer ports are used [1]. The ideal theoretical values for the coupling coefficients are $C_{b a r}=0.71$ and $C_{x}=-0.21$ [9]. This implies that if $E_{1}$ and $E_{2}$ are equal and opposite, the coupler ideally would behave as a $100 \%$ reflector for both lasers.

As indicated in Fig. 1 the reference frequency for the coupled laser is $\omega_{0}$, which we assume to be a conveniently chosen frequency, such as to provide adequate numerical accuracy and convergence speed. A particular choice for $\omega_{0}$ will be postponed until sec. 4. The only known and given frequencies are $\omega_{1}$ and $\omega_{2}$, the respective operating frequencies of the constituent lasers 1 and 2 when they operate without coupling; i.e. with $C_{x}=0$ and at their thresholds.

After one roundtrip in laser 1 we can write

$$
E_{1}\left(t+\tau_{1}\right)=e^{2 i k_{1} L_{1}} r^{2}\left[E_{1}(t) C_{b a r}+E_{2}(t) C_{x}\right]
$$

where $r$ is the reflection coefficient (assumed real) at the left and right end of each laser, $k_{j} \equiv k_{j}{ }^{\prime}+$ $i k_{j}$ " is the wavenumber and $\tau_{j}$ the roundtrip time in laser $j$. Hence we can write

$$
\dot{E}_{1}(t)=\frac{E_{1}\left(t+\tau_{1}\right)-E_{1}(t)}{\tau_{1}} \quad=\frac{\left(e^{2 i k_{1} L_{1}} r^{2} C_{b a r}-1\right)}{\tau_{1}} E_{1}(t)+\frac{C_{x} r^{2} e^{2 i k_{1} L_{1}}}{\tau_{1}} E_{2}(t),
$$

and a similar equation for $E_{2}$ driven by $E_{1}$.

We introduce $\left(v_{g, j}\right.$ is the group velocity and $N_{j}$ the inversion in laser $j$ )

$$
\xi_{j} \equiv-2 v_{g, j} \frac{\partial k^{\prime \prime}}{\partial N_{j}} ; \alpha_{j} \equiv 2 v_{g, j} \frac{\partial k^{\prime}{ }_{j}}{\partial N_{j}} / \xi_{j}
$$

the gain coefficient and linewidth-enhancement factor of laser $j$, respectively, and choose the

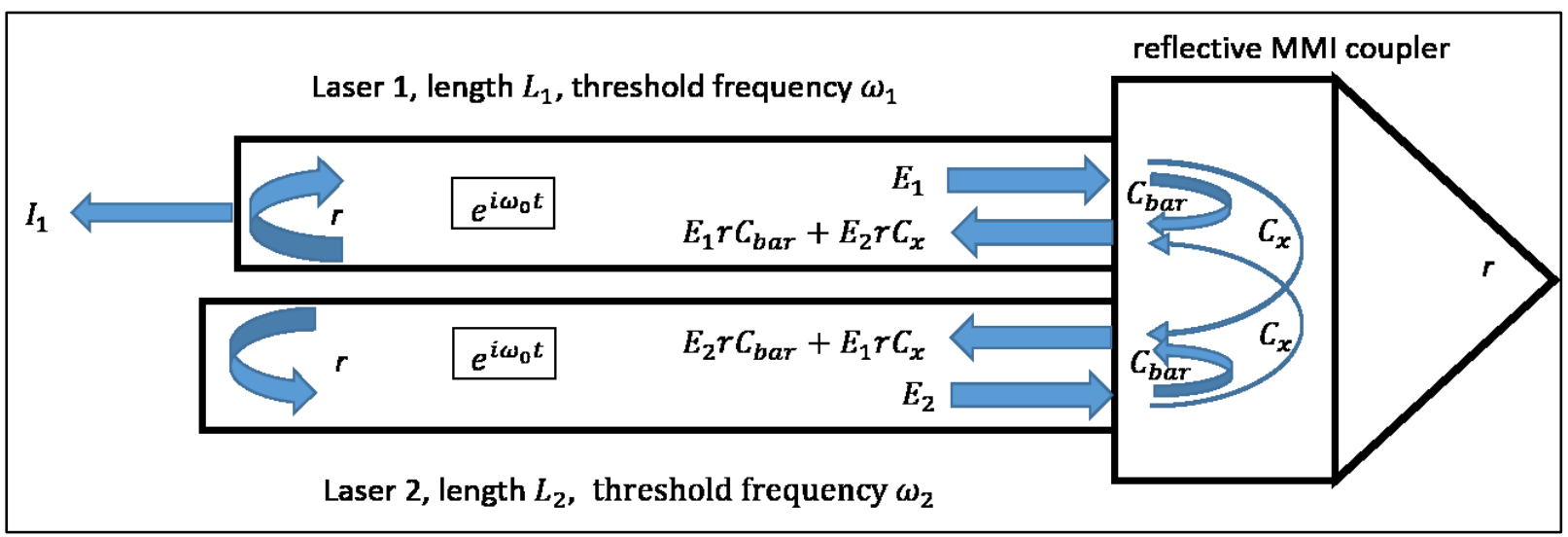

Fig. 1. Sketch of two FP-lasers coupled by a reflective MMI coupler with designed coupling parameters $C_{b a r}$ and $C_{x}$. The slowly-varying amplitudes are relative to the exponential time factor as indicated, with $\omega_{0}$ a conveniently chosen frequency. $\omega_{1}, \omega_{2}$ are the frequencies of the isolated lasers, i.e. when $C_{x}=0$. 
threshold condition of each isolated laser (i.e. no coupling) as $N_{j}=0$ and corresponding reference frequency $\omega_{j}$. In that case, we have by definition

$$
r^{2} C_{b a r} e^{-2 k^{\prime \prime}\left(N_{j}=0\right) L_{j}+2 i k^{\prime}{ }_{j}\left(N_{j}=0\right) L_{j}}=e^{i \omega_{j 0} \tau_{j}},
$$

where $\omega_{j 0} \equiv \omega_{j}-\omega_{0}$ for $j=1,2$. Hence we can write, using (3) and $\tau_{j} \equiv 2 L_{j} / v_{g, j}$,

$$
e^{2 i k_{j} L_{j}}=\frac{e^{\frac{1}{2}\left(1+i \alpha_{j}\right) \xi_{j} N_{j} \tau_{j}+i \omega_{j 0} \tau_{j}}}{r^{2} C_{b a r}}
$$

with $N_{j}$ the e-h populations relative to their threshold values in the respective isolated lasers. This allows to express the first term in the last line of (2) in first-order approximation as

$$
\frac{\left(e^{2 i k_{1} L_{1}} r^{2} C_{b a r}-1\right)}{\tau_{1}} E_{1}(t) \cong i \omega_{10} E_{1}(t)+\frac{1}{2}\left(1+i \alpha_{1}\right) \xi_{1} N_{1} E_{1}(t) .
$$

Hence, (2) can be written as

$$
\dot{E}_{1}(t)=i \omega_{10} E_{1}(t)+\frac{1}{2}\left(1+i \alpha_{1}\right) \xi_{1} N_{1} E_{1}(t)+\frac{e^{\frac{1}{2}\left(1+i \alpha_{1}\right) \xi_{1} N_{1} \tau_{1}}}{C_{b a r} \tau_{1}} C_{x} e^{i \omega_{10} \tau_{1}} E_{2}(t)
$$

Similarly for $E_{2}(t)$ we can write,

$$
\dot{E}_{2}(t)=i \omega_{20} E_{2}(t)+\frac{1}{2}\left(1+i \alpha_{2}\right) \xi_{2} N_{2} E_{2}(t)+\frac{e^{\frac{1}{2}\left(1+i \alpha_{2}\right) \xi_{2} N_{2} \tau_{2}}}{C_{b a r} \tau_{2}} C_{x} e^{i \omega_{20} \tau_{2}} E_{1}(t)
$$

and obtain for the weakly time-dependent laser amplitudes

$$
\begin{aligned}
& \dot{E_{1}}(t)=i \omega_{10} E_{1}(t)+\frac{1}{2}\left(1+i \alpha_{1}\right) \xi_{1} N_{1} E_{1}(t)+\kappa_{1} E_{2}(t), \\
& \dot{E}_{2}(t)=i \omega_{20} E_{2}(t)+\frac{1}{2}\left(1+i \alpha_{2}\right) \xi_{2} N_{2} E_{2}(t)+\kappa_{2} E_{1}(t),
\end{aligned}
$$

Here, $\kappa_{j}(j=1,2)$, are the (effective) coupling coefficients,

$$
\kappa_{j}=\frac{e^{\frac{1}{2}\left(1+i \alpha_{j}\right) \xi_{j} N_{j} \tau_{j}}}{C_{b a r} \tau_{j}} C_{x} e^{i \omega_{j 0} \tau_{j}} \equiv-\left|\kappa_{j}\right| e^{i \theta_{j}}
$$

The minus sign in the right-hand side (r.h.s.) of (10) accounts for the negative value of $C_{x} / C_{b a r}$ and

$$
\theta_{j} \equiv \frac{1}{2} \alpha_{j} \xi_{j} N_{j} \tau_{j}+\omega_{j 0} \tau_{j},\left|\kappa_{j}\right| \equiv \frac{e^{\frac{1}{2} \xi_{j} N_{j} \tau_{j}}}{C_{b a r} \tau_{j}}\left|C_{x}\right|
$$

Note that $\theta_{j}$ and $\left|\kappa_{j}\right|$ are functions of $N_{j}(j=1,2)$. As we expect that due to the coupling the intensities in both lasers will increase, the steady-state values of $\left\{N_{j}\right\}$ will assume appreciable negative values different from 0 . This causes the locking of the coupled lasers to become a complex nonlinear problem as was recognized too in earlier treatments of coupled lasers [7,8], but there the role of $\alpha$-induced chirping was not explicitly dealt with. This inversion-dependent effective coupling is often disregarded in delay-coupled laser systems, see for instance [10]. 
Eqs. (9) are complemented by the rate equations for the numbers of e.h.-pairs in the respective active regions

$$
\begin{aligned}
& \dot{N}_{1}=\Delta J_{1}-\frac{N_{1}}{T}-\xi N_{1} P_{1}-\Gamma_{1} P_{1}, \\
& \dot{N}_{2}=\Delta J_{2}-\frac{N_{2}}{T}-\xi N_{2} P_{2}-\Gamma_{2} P_{2},
\end{aligned}
$$

where, consistent with the choice made below (5), $\Delta J_{j}$ is the injection current w.r.t. the threshold current (i.e. when each laser is on its own, that is, without any coupling; $\Delta J_{j}$ in units $s^{-1}$ ), $T$ is the spontaneous recombination lifetime of e.h.-pairs and

$$
\Gamma_{j}=\frac{2}{\tau_{j}}\left(1-r^{2} C_{b a r}\right)
$$

is the photon decay rate in isolated laser $j$. The thresholds are defined for each isolated laser, i.e. for $C_{x}=0$. The coupled equations (9) and (12) are the rate equations for the coupled-cavity laser.

\section{Steady-state analysis}

To facilitate the steady-state analysis, it is convenient to formulate equations for the intensities and phases $\left(E_{j}=\sqrt{P_{j}} e^{i \varphi_{j}}\right)$ :

$$
\begin{aligned}
& \dot{P_{1}}(t)=\xi_{1} N_{1} P_{1}(t)-2\left|\kappa_{1}\right| \sqrt{P_{1} P_{2}} \cos \left(\theta_{1}+\varphi_{21}\right) ; \\
& \dot{P_{2}}(t)=\xi_{2} N_{2} P_{2}(t)-2\left|\kappa_{2}\right| \sqrt{P_{1} P_{2}} \cos \left(\theta_{2}-\varphi_{21}\right) ; \\
& \dot{\varphi}_{1}(t)=\omega_{10}+\frac{1}{2} \alpha_{1} \xi_{1} N_{1}-\left|\kappa_{1}\right| \sqrt{\frac{P_{2}}{P_{1}}} \sin \left(\theta_{1}+\varphi_{21}\right) ; \\
& \dot{\varphi}_{2}(t)=\omega_{20}+\frac{1}{2} \alpha_{2} \xi_{2} N_{2}-\left|\kappa_{2}\right| \sqrt{\frac{P_{1}}{P_{2}}} \sin \left(\theta_{2}-\varphi_{21}\right) .
\end{aligned}
$$

In (14) and (15) we have introduced the phase difference

$$
\varphi_{21} \equiv \varphi_{2}-\varphi_{1}
$$

We study the situation where the two lasers are locked to each other, meaning that they operate both on the same frequency $\omega_{o p}$. If such steady state exists, it follows from the first equation in (15) that

$$
\omega_{o p}=\omega_{10}+\frac{1}{2} \alpha_{1} \xi_{1} N_{1}-\left|\kappa_{1}\right| \sqrt{\frac{P_{2}}{P_{1}}} \sin \left(\theta_{1}+\Phi_{21}\right)
$$

with $\Phi_{21}$ the steady-state locked (time-independent) value of $\varphi_{21}$. Alternatively, using the second equation (15), we can also find in the locked state

$$
\omega_{o p}=\omega_{20}+\frac{1}{2} \alpha_{2} \xi_{2} N_{2}-\left|\kappa_{2}\right| \sqrt{\frac{P_{1}}{P_{2}}} \sin \left(\theta_{2}-\Phi_{21}\right)
$$

We can combine eqs. (15) as (use $\left.\omega_{20}-\omega_{10}=\omega_{21}\right)$

$$
\dot{\varphi_{21}}=\omega_{21}+\frac{1}{2}\left(\alpha_{2} \xi_{2} N_{2}-\alpha_{1} \xi_{1} N_{1}\right)-\left|\kappa_{2}\right| \sqrt{\frac{P_{1}}{P_{2}}} \sin \left(\theta_{2}-\varphi_{21}\right)+\left|\kappa_{1}\right| \sqrt{\frac{P_{2}}{P_{1}}} \sin \left(\theta_{1}+\varphi_{21}\right)
$$


Eq. (19) describes the locking dynamics; in the steady state the left-hand side vanishes and the resulting equation is equivalent to (17) and (18). The steady-state solutions of (14) satisfy

$$
\sqrt{\frac{P_{1}}{P_{2}}}=\frac{2\left|\kappa_{1}\right| \cos \left(\theta_{1}+\varphi_{21}\right)}{\xi_{1} N_{1}} ; \quad \sqrt{\frac{P_{2}}{P_{1}}}=\frac{2\left|\kappa_{2}\right| \cos \left(\theta_{2}-\varphi_{21}\right)}{\xi_{2} N_{2}},
$$

hence, by substitution of (20) in (15), we can write,

$$
\begin{aligned}
& \dot{\varphi}_{1}=\omega_{10}-\frac{\left|\kappa_{1}\right|}{\epsilon} \sqrt{1+\alpha_{1}^{2}} \sin \left(\theta_{1}+\varphi_{21}-\operatorname{Atan} \alpha_{1}\right), \\
& \dot{\varphi}_{2}=\omega_{20}-\epsilon\left|\kappa_{2}\right| \sqrt{1+\alpha_{2}^{2}} \sin \left(\theta_{2}-\varphi_{21}-\operatorname{Atan} \alpha_{2}\right) .
\end{aligned}
$$

Here we introduced the notation

$$
\epsilon \equiv \sqrt{P_{1} / P_{2}}
$$

From (21) we obtain, as an equivalent alternative to (19),

$$
\begin{aligned}
\varphi_{21}^{\cdot} & =\omega_{21}-\epsilon\left|\kappa_{2}\right| \sqrt{1+\alpha_{2}^{2}} \sin \left(\theta_{2}-\varphi_{21}-\operatorname{Atan} \alpha_{2}\right) \\
& +\frac{\left|\kappa_{1}\right|}{\epsilon} \sqrt{1+\alpha_{1}^{2}} \sin \left(\theta_{1}+\varphi_{21}-\operatorname{Atan} \alpha_{1}\right),
\end{aligned}
$$

where $\omega_{21}=\omega_{2}-\omega_{1}$. This can also be written, by combining the two sinus functions in the righthand side of (23), as

$$
\varphi_{21}^{\cdot}=\omega_{21}+\sqrt{C^{2}+D^{2}} \sin \left(\varphi_{21}+\Psi\right)
$$

with

$$
\begin{aligned}
& \Psi=\operatorname{Atan}\left(\frac{C}{D}\right) \\
& C=-\epsilon\left|\kappa_{2}\right| \sqrt{1+\alpha_{2}^{2}} \sin \left(\theta_{2}-\operatorname{Atan} \alpha_{2}\right)+\frac{\left|\kappa_{1}\right|}{\epsilon} \sqrt{1+\alpha_{1}^{2}} \sin \left(\theta_{1}-\operatorname{Atan} \alpha_{1}\right) \\
& D=\epsilon\left|\kappa_{2}\right| \sqrt{1+\alpha_{2}^{2}} \cos \left(\theta_{2}-\operatorname{Atan} \alpha_{2}\right)+\frac{\left|\kappa_{1}\right|}{\epsilon} \sqrt{1+\alpha_{1}^{2}} \cos \left(\theta_{1}-\operatorname{Atan} \alpha_{1}\right)
\end{aligned}
$$

From the steady-state solutions of (12) we derive

$$
P_{j}=\frac{\Delta J_{j}-\frac{N_{j}}{T}}{\Gamma_{j}+\xi_{j} N_{j}}
$$

and this can be used in (22) to evaluate $\epsilon$. If the locking condition

$$
\omega_{21} \leq \sqrt{C^{2}+D^{2}}
$$

is satisfied, the stable steady-state solution of (24) is given by

$$
\Phi_{21}=A \sin \left(\frac{\omega_{21}}{\sqrt{C^{2}+D^{2}}}\right)-\Psi+\pi
$$


It is possible to adjust the detuning in such a way that the "optimal" coupling situation with $\Phi_{21}=\pi$ is realized. This optimized detuning is given by

$$
\left.\omega_{21}\right|_{\text {opt }}=C \text {. }
$$

It is noted that "optimal" coupling means the lasers operate in perfect anti-phase coupling, a situation of maximized intensities $P_{1}$ and $P_{2}$.

In view of the numerical strategy to be described in the next section, it is very helpful to derive an expression for the inversions in terms of the phase difference $\Phi_{21}$ and the ratio $\epsilon$ of amplitudes in the locked situation. Combining the defining equations (11) with the locking equations (20) we can express, in good approximation (truncated after four iterations),

$$
N_{j}=\frac{2 A_{j}}{\xi_{j} \tau_{j}} \exp \left[A_{j} \exp \left[A_{j} \exp \left[A_{j} \exp \left[A_{j}\right]\right]\right]\right]
$$

with

$$
A_{1} \equiv \frac{C_{x}}{C_{\text {bar }} \epsilon} \cos \left(\theta_{1}+\Phi_{21}\right) ; A_{2} \equiv \frac{C_{x} \epsilon}{C_{b a r}} \cos \left(\theta_{2}-\Phi_{21}\right)
$$

Finally, we need an expression for the output intensity $I_{1}$ leaving laser 1 at the left facet. From Fig. 1 and using eq. 5 for $j=1$, it can be deduced that

$$
I_{1}=\left(1-r^{2}\right) C_{b a r} e^{-\frac{1}{2} \xi_{1} N_{1} \tau_{1}} P_{1}
$$

This concludes the formulation of the equations describing the stable steady-state operation in terms of observable quantities of the coupled-cavity laser.

\section{Numerical results}

Numerical results have been obtained for parameter values given in Table 1. A Mathematica code was used to solve the coupled equations (20), (26), (28) and (30) by iteration. This procedure worked well by choosing $\omega_{0}$ such that

$$
\omega_{10} \equiv \omega_{1}-\omega_{0}=-\frac{1}{2} \omega_{21}-\frac{1}{4}\left(\alpha_{1} \xi_{1} N_{1}+\alpha_{2} \xi_{2} N_{2}\right)
$$

This choice keeps $\omega_{j 0}$ as small as possible for all values of $\alpha_{j}$ and minimizes the error made in the first-order approximation in the transition from (6) to (7). Numerical instabilities only started to occur for $\alpha$ values larger than $\sim 30$. The frequency range of locking was seen to be $\sim 30$ to $40 \times$ $10^{9} \mathrm{Rad} / \mathrm{s}$ for $\alpha_{j}=0$ all the way up to $\alpha_{j}=30$.

The following numerical iteration scheme was applied for each given value of the detuning $\omega_{21}$ : In the first step we start with initial values $\theta_{j}=0, \Phi_{21}=\pi$ and $\epsilon=1$. With these values $N_{1}$ and $N_{2}$ are calculated using (31) and (32). Then we evaluate $\left|\kappa_{j}\right|, \theta_{j}, P_{j}$ and update $\epsilon$, using (11), (26) and (22), where after a new value for $\Phi_{21}$ is obtained using (25) and (28). This procedure is repeated until convergence is reached, which is normally the case after less than 100 iteration steps. The procedure is also terminated when the locking criterion (27) is violated. 
Table 1: Parameter values for the coupled-cavity laser considered

\begin{tabular}{ccl}
\hline Symbol & Value & Name \\
$\alpha_{j}$ & 2.5 & Linewidth enhancement parameter \\
$\xi$ & $1.0 \times 10^{3} \mathrm{~s}^{-1}$ & Linear gain coefficient (at threshold) \\
$\tau_{1}$ & $2.40 \times 10^{-11} \mathrm{~s}$ & Roundtrip time FP cavity 1 \\
$\tau_{2}$ & $2.52 \times 10^{-11} \mathrm{~s}$ & Roundtrip time FP cavity 2 \\
$T$ & $1.0 \times 10^{-9} \mathrm{~s}$ & e-h- lifetime for spontaneous recombination \\
$\Gamma_{1}$ & $5.27 \times 10^{10} \mathrm{~s}^{-1}$ & Photon decay rate of cavity 1 \\
$\Gamma_{2}$ & $5.02 \times 10^{10} \mathrm{~s}^{-1}$ & Photon decay rate of cavity 2 \\
$C_{b a r}$ & 0.75 & Self-coupling coefficient of MMI-reflector \\
$C_{x}$ & -0.18 & Cross-coupling coefficient of MMI-reflector \\
$r$ & 0.7 & Reflection coefficient of mirrors \\
$J_{t h r, j}(\mathrm{j}=1,2)$ & $1.1 \times 10^{17} \mathrm{~s}^{-1}$ & Threshold current \\
& $(\sim 18 \mathrm{~mA})$ & \\
\hline
\end{tabular}

The values for $C_{\mathrm{bar}}$ and $C_{\mathrm{x}}$ given in Table 1 are not the "ideal" theoretical values for the $3 \times 3$ reflective MMI coupler [1,9], but yield best agreement for $\alpha_{1}=\alpha_{2}=2.5$ with the output intensity curve shapes measured in [1]. In reality, values of the MMI- coupling parameters deviating from their theoretical values [9], i.e. $C_{\mathrm{x}}=-0.21, C_{\mathrm{bar}}=0.79$, are not unlikely, because of imperfect imaging in the MMI-reflective coupler. We believe that these deviations are well within the growth specifications. It was numerically verified that replacing $\alpha_{j}$ by $-\alpha_{j}$ yields the same results as replacing $\omega_{21}$ by $-\omega_{21}$. In Figs 2-4 we define the pump strength $p_{j}$ of laser $j$ as

$$
p_{j} \equiv \frac{\Delta J_{j}}{J_{t h r, j}}
$$

where $J_{t h r, j}$ is the threshold injection current of laser $j$.

In Fig.2 numerical results are presented for the output intensity $I_{1}$ and the operation frequency $\omega_{o p 1} \equiv \omega_{o p}-\omega_{1}$ showing the locking range extending from $\omega_{21}=-16$ to +16 $\mathrm{Grad} / \mathrm{s}$, for a case of strongly asymmetric pumping. Outside the locking range, the intensity and operation frequency are set to zero. In this region the coupled system is not able to operate in steady state. In reality, the coupled laser would have jumped here to a neighboring combination of modes, possibly assisted by small adjustments of the constituent laser frequencies (by phase shifting one laser, for instance).

Generally, the shapes of the numerical output-intensity curves (see Fig. 4) hardly depend on the value of $\alpha$. On the other hand, the operation frequency in locked operation does depend sensitively on $\alpha$. This is a consequence of the inversions in the coupled laser "clamping" at negative values. For the symmetrically pumped case of Fig. 3 the clamping is demonstrated by the nearly constant value of $\omega_{o p 1}$ slightly smaller than $-2 \times 10^{10} \mathrm{Rad} / \mathrm{s}$, independent of the pump strength. From the first equation in (21) we find

$$
\omega_{o p 1}=-\frac{\left|\kappa_{1}\right|}{\epsilon} \sqrt{1+\alpha_{1}^{2}} \sin \left(\theta_{1}+\varphi_{21}-\operatorname{Atan} \alpha_{1}\right),
$$




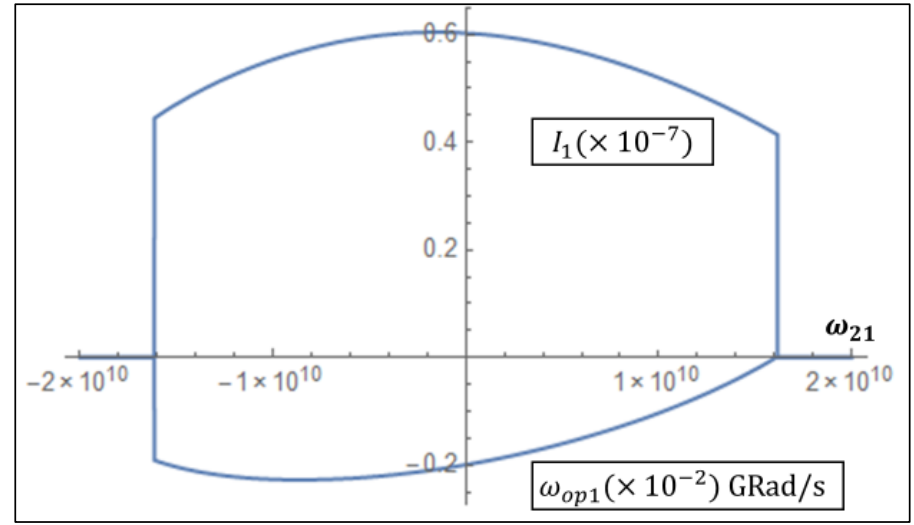

Fig. 2. Calculated intensity $I_{1}\left(\times 10^{-7}\right)$ and operation-frequency shift $\omega_{o p 1}\left(\times 10^{-11}\right)$ versus detuning $\omega_{21} \equiv \omega_{2}-\omega_{1}$ within the locking range. The pump strengths are $p 1=1, p 2=4$.

which for optimized tuning $\left(\Phi_{21}=\pi\right)$, symmetric pumping $\left(p_{1}=p_{2}\right)$ and $\epsilon \approx 1$ approximately equals

$$
\omega_{o p 1} \cong\left|\kappa_{1}\right| \sqrt{1+\alpha_{j}^{2}} \sin \left(\theta_{1}-\operatorname{Atan} \alpha_{j}\right)=-\alpha_{j}\left|\kappa_{1}\right| \sim-2.2 \times 10^{10} \mathrm{Rad} / \mathrm{s},
$$

in agreement with the value found in Fig. 3. Also indicated in Fig. 3 is the value for the detuning $\omega_{21} l_{\text {opt }}$, see (29), for optimized coupling. It lies slightly below the horizontal axis. Due to symmetric pumping, the long laser (i.e. laser 2) has slightly higher weight than the shorter laser 1 in determining the optimum condition.

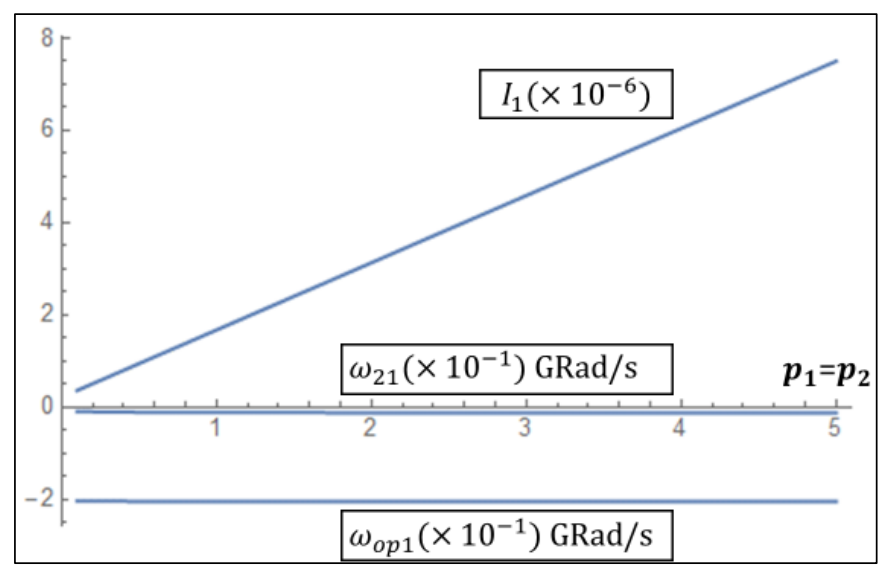

Fig. 3. Output intensity $I_{1}$ and operation-frequency shift $\omega_{o p 1}$ versus $p_{1}=p_{2}$ under optimized detuning condition. Note that the effective threshold of the laser is below zero. This is because under equal intensity condition the combined laser system has reduced roundtrip loss, compared to the uncoupled situation.

In Fig. 4 a series of output intensity curves is presented assuming optimized tuning conditions as explained below (28). The pump strength values are chosen such as to match those in the measured curves of fig.5 in ref. [1]. The curves are in good qualitative and quantitative (proportional) agreement. 


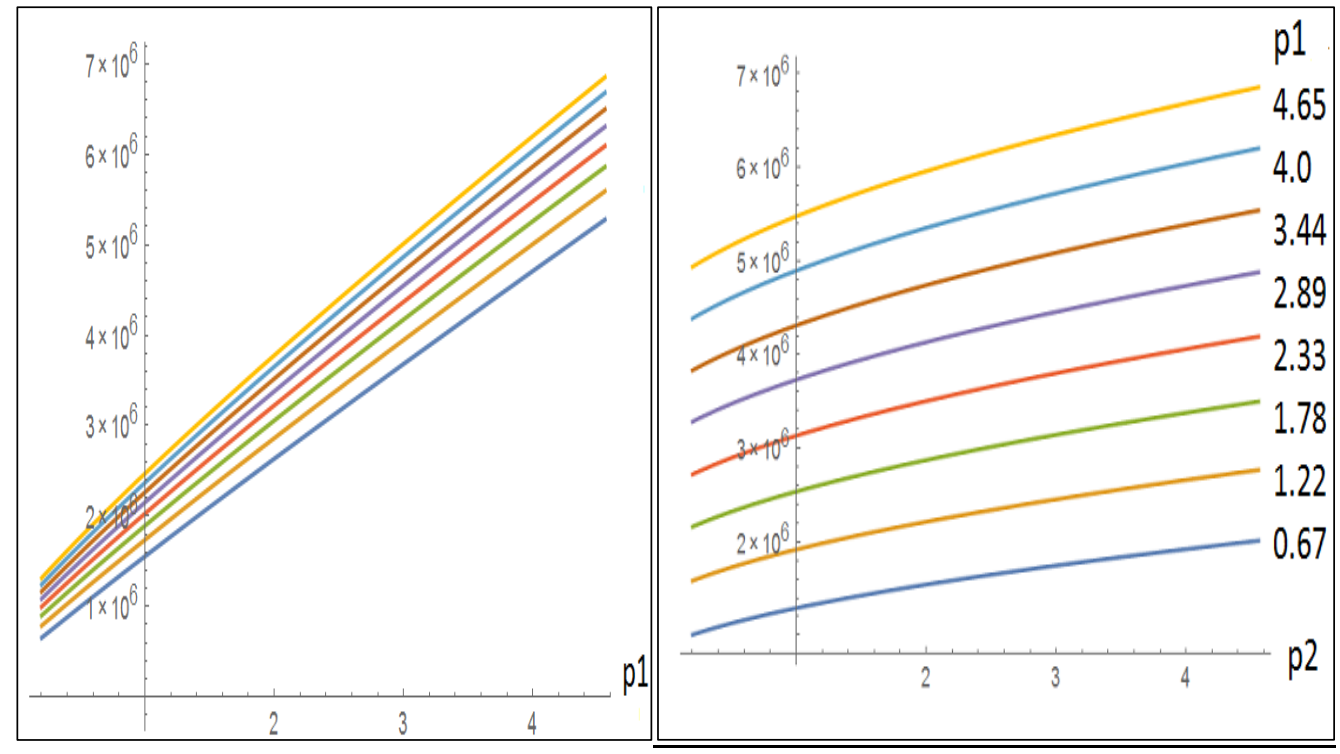

Fig. 4. Left: Intensity $I_{1}$ versus $p_{1}$, at fixed $p_{2}=\{0.67,1.22,1.78,2.33,2.89,3.44,4.0,4.65\}$. Right: intensity $I_{1}$ versus $p_{2}$, with fixed $p_{1}=$ \{same values $\}$; optimized detuning assumed. The shapes of the curves are not very sensitive to the precise value of $\alpha$. The values for $C_{\mathrm{bar}}$ and $C_{\mathrm{x}}$ (see table 1) were taken so as to optimize the qualitative and quantitative (proportional) agreement with the measured curves (Fig. 5 in [1]).

\section{Conclusions}

We have presented a rate-equation theory which adequately describes the locking of two semiconductor Fabry-Pérot laser cavities coupled by an anti-phase MMI coupler. It is shown that due to the strong mutual injection the effective coupling coefficients depend on the resulting inversions of the individual lasers, where the latter in turn are strongly perturbed by the coupling. Thus, a steady state in which the two lasers are locked in frequency depends on the effective coupling, which itself depends on the respective inversions. This requires a self-consistent solution method, which is realized by iteration.

We have shown the existence of detuning intervals where the coupled-laser system locks to a single operation frequency, for all practical values of the linewidth-enhancement parameter. The width of these locking intervals is around $\sim 35 \mathrm{Grad} / \mathrm{s}$ almost independent of $\alpha_{j}$. A sizeable detuning interval of locking is important, as it allows proper fine tuning of the laser system, a feature fully employed in ref. [1].

It was found in general that numerical convergence to a locked situation was easy to achieve, typically in $\sim 50$ iterations. Considering all analytical and numerical results for the locked operation of the CCL here presented, we found that results with $\alpha$ set equal to 2.5 and $C_{b a r}=0.75, C_{x}=-0.18$ provide a good match with the experimental results of ref. [1], both qualitatively and, in proportion, quantitatively, although dependence of the output intensity curves on $\alpha$ is rather weak. Values somewhat different from the "ideal" theoretical values [10] for the bar and cross coupling coefficients, i.e. 0.79 and -0.21 , respectively, can indeed be expected as a result of slight changes in growth specifications.

\section{Acknowledgment}

The author wishes to thank Jan Mink (VTEC) and Weiming Zhao (Eindhoven University of Technology) for their stimulating interest. 


\section{References}

[1] D. D'Agostino, D. Lenstra, H. P. M. M. Ambrosius, M. K. Smit, Coupled cavity laser based on anti-resonant imaging via multimode interference, Optics Letters. 40 (2015) 653.

[2] L. A. Coldren, T. L. Koch, Analysis and design of coupled-cavity lasers, I and II, IEEE Journal of Quantum Electron. 20 (1984) 659-682

[3] W. Streiffer, D. Yevick, T.L. Paoli, R.D Burnham, An analysis of cleaved coupled-cavity lasers, IEEE Journal of Quantum Electronics. QE-20 (1984) 754-764.

[4] Jian-Jun He, Dekan Liu, Wavelength switchable semiconductor laser using half-wave Vcoupled cavities, Optics Express. 16 (2008) 3896-3911.

[5] K.J. Ebeling, L.A. Coldren, Analysis of multielement semiconductor lasers, J. Appl. Phys. 54 (1983) 2962-2969.

[6] F.D. Khan, D.T. Cassidy, Widely tunable coupled-cavity semiconductor laser, Applied Optics. 48 (2009) 3809-3817.

[7] D. Marcuse, Tien-Pei Lee, Rate equation model of a coupled-cavity laser, IEEE Journal of Quantum Electron. 20 (1984) 166-176

[8] R.J. Lang, A. Yariv, Local-field rate equations for coupled optical resonators, Phys. Rev. A 34 (1986) 2038-2043.

[9] D. Lenstra, 3 x 3 Multi-mode interference coupler theory. DOI: $10.13140 /$ RG.2.1.1015.0163 (2014).

[10] J. Mulet, C. Mirasso, T. Heil, I. Fischer, Synchronization scenario of two distant mutually coupled semiconductor lasers, J. Opt. B: Quantum Semiclass. Opt. 6 (2004) 97-105 